\title{
Three-Dimensional Image Analysis to Quantify the Temporo-Spacial Expression of Cellular Receptors
}

\author{
Sarmed Al-Samerria and Ghanim Almahbobi \\ School of Biomedical Sciences, CHIRI, Curtin University, Perth, WA, Australia \\ Email:sarmed.alsamerria@postgrad.curtin.edu.aułg.almahbobi@curtin.edu.au
}

\begin{abstract}
-0varian folliculogenesis is primarily controlled by the action of gonadotropins namely follicle stimulating hormone (FSH) and luteinizing hormone (LH). Several reports indicated that the process of initial recruitment of primordial follicles to the growing follicles is not gonadotropin-dependent but Bone morphogenetic protein (BMP)-dependent. However, this has not been unequivocally confirmed. The aim of this study was to investigate the temporo-spacial protein expression of the $B M P$ receptors 1B (BMPR1b), FSHR and LHR in several stages of follicle development. While the localization of all receptors was found in granulosa cell membrane of the follicles the temporal expression was varied. BMPR1b was expressed in all follicle stages, FSHR was detected in primary follicles onward and LHR was absent in both primordial and primary follicles but appeared in later stages. Quantitative analysis based on the intensity of fluorescent signals showed that the expression of BMPR1b, FSHR and LHR significantly $(p<0.001 p<0.0001 p<0.0001$ respectively) increased with follicular development. We have concluded that the combination of sensitive immunofluorescence labeling and computerized 3D image analysis proves efficient tools for in situ detection and quantification of the expression of small amount of protein in a complex tissue structure.
\end{abstract}

Index Terms -folliculogenesis, Bone morphogenetic protein, follicle stimulating hormone (FSH), luteinizing hormone (LH), 3D image analysis

\section{INTRODUCTION}

Ovarian folliculogenesis is the basis of the entire ovarian function including the production of mature oocytes ready for fertilization and the sexual hormones required for the development of female phenotypes. This process entails a series of chronological steps in which a growing follicle either matures to ovulation or dies by apoptosis [1]. The primordial follicle considered as the constructional units of female ovary. The initial transition of primordial follicles to growing primary follicles is the key limiting step in preserving or depletion of the female fertility reserve. It is well established that folliculogenesis is regulated by the gonadotropins FSH and $\mathrm{LH}$, which are secreted by the pituitary gland [2]. In addition, complex autocrine and paracrine actions of several intra-ovarian factors such as the BMPs influence the action of gonadotropins [3]. For instance, it is now believed that

Manuscript received July 1, 2013; revised September 15, 2013. the initial stage of follicle recruitment and development of primordial to primary follicles is independent of the gonadotropins [4] but rather associated with a specific spatial and temporal pattern of BMP expression [5].

However, such interplay between the gonadotropins and BMPs particularly in the initial recruitment of primordial

Follicles remain inconclusive. Because of the complex nature of the ovarian structure and the dynamics of folliculogenesis, most of the studies in relation to this issue have been conducted in vitro using molecular and biochemical approaches [6] or in situ microscopic analysis using manual subjective methods 7 ].

The use of new non-bias computerized technology in biomedical research has increased in the past decades. The computer-based method was introduced to provide accurate and reliable quantitative information as well as a non-time consuming tool [8]. This technology will eventually replace the conventional manual and subjective methods. In attempt to clarify the regulation of initial folliculogenesis; the present study demonstrates the interaction between BMP and gonadotropin signaling systems, using immunofluorescence detection and 3D image analysis of cell-membrane bound receptors of BMPs, FSH and $\mathrm{LH}$ in developing follicles of sheep ovaries.

\section{MATERIALS AND METHODS}

\section{A. Sample Collection and Histology}

Sheep ovaries were collected from local abattoir and divided into 2 groups. One group was snap-frozen in liquid nitrogen and stored in $-80^{\circ} \mathrm{C}$ for further usage. The second group was fixed in $10 \%$ Neutral Buffered Formalin (NPF) and processed using Tissue-Tek VIP automatic tissue processor and embedded into paraffin wax. Tissue sections of $5 \mu \mathrm{m}$ were cut, placed onto super frost slides (HD scientific supplies Pty Ltd, Australia) and stained with Hematoxylin and Eosin for morphological study to identify the different stages of follicular development.

\section{B. Immuonoflourescent Labeling of BMPR1b, FSHR and $L H R$}

Frozen tissues were partially embedded in OCT and $10 \mu \mathrm{m}$ sections were prepared using a Cryostat (Carl Zeiss, 
Sydney, NSW, Australia) and fixed in 4\% paraformaldehyde at $4^{\circ} \mathrm{C}$ for $7 \mathrm{~min}$. Indirect immunofluorescence labeling was performed as previously reported [9]. In brief, $4 \mu \mathrm{g} / \mathrm{ml}$ of the primary antibodies was applied overnight at $4^{\circ} \mathrm{C}$ in humidified chamber. The antibodies are (monoclonal) goat antiBMPR1b (sc-5679), goat anti-FSHR (sc-7798) and goat anti-LHR (sc-26341), all from Santa Cruz Biotechnology, Santa Cruz, CA, USA. After a serial of washing a donkey Anti-goat second antibody conjugated with Alexa 488 (Molecular Probes, Australia) was added for $45 \mathrm{~min}$ in dark humidified chamber. Negative controls were performed by omitting the second antibodies for the presence of auto-fluorescent signals and non-specific binding. The sections were mounted using anti-Fade aqueous mounting medium containing 40, 60-diamidino2-phenylindole (DAPI; Molecular Probes, NSW, Australia).

The sections were examined by Carl Zeiss semiconfocal fluorescent microscope equipped with CarlZeiss Digital Camera (200M Axiovert; Carl Zeiss, Sydney, NSW, Australia) and the images were captured using AsioVision 4.2.8 image analyzer software.

\section{3D Image Analysis and Immuonoflourescent Intensity Quantification}

For image acquiring, the exposure time was adjusted using control sections incubated with pre-immune serum in order to subtract auto-fluorescent and non-specific binding background. Surplus fluorescent signals, appeared after such subtraction, were considered specific binding, which were subjected for quantification study. Z-stalks of $10 \times 1 \mu \mathrm{m}$ optical frames/sections, generated from the $10 \mu \mathrm{m}$ thick physical sections, were captured and compiled to generate $3 \mathrm{D}$ images. The $3 \mathrm{D}$ images were used to quantify the intensity of immuonoflourescent signals in the entire $10 \mu \mathrm{m}$ sections (Fig. 1).

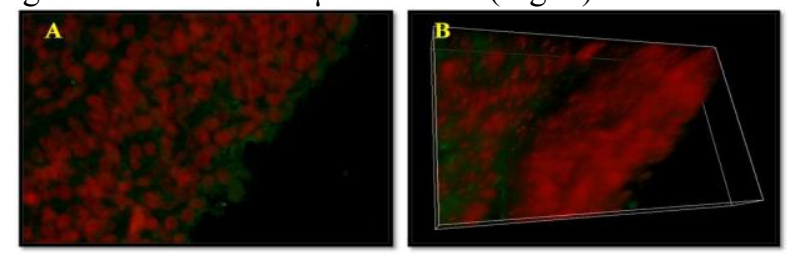

Figure 1. Granulosa cells from pre-ovulary follicles A. 2D image to determine the Localization and receptor expression B. 3D image for immuonoflourescent quantification. X40

\section{Statistical Analysis}

Statistical analysis was performed using prism version 6 (Graph Pad Software, La Jolla, CA, USA). The results were analyzed using the mean and standard deviation of BMPR-1b, FSHR and LHR expression in four stages of follicular development and expressed as mean pixel $/ \mu \mathrm{m}^{2}$ \pm SEM.

\section{RESULTS}

\section{A. Morphological Assessment}

Four stages of follicular development have been identified and subjected to this study (Fig. 2A-D). Primordial follicles (Fig. 2A) consist of a primary oocyte surrounded by few spindle-shaped cells, granulosa cells (GCs). The primary follicle (Fig. 2B) initially consists of a primary oocyte surrounded by a complete layer of cuboidal GCs. The zona pellucid (ZP), a thick layer composed of glycoprotein and acid proteoglycans, forms between the oocyte and GCs. Once GCs proliferate and arrange into multiple layers the secondary follicle is formed (Fig. 2C). The Graafian follicle is characterized by a large, fluid-filled antrum and an eccentric oocyte (Fig. 2D).

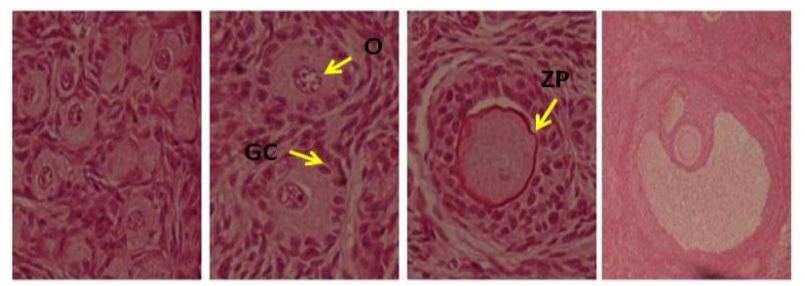

Figure 2. Four stages of follicle development A) Primordial follicle B) Primary follicle C) Secondary follicle D) pre-ovulatory follicle.

\section{B. Immuonoflourescent Localization of BMP1b, FSHR and $L H R$}

Immunofluorescence microscopy revealed that the intensity of a positive immunolabelling of the membranebound receptors varies based on the stage of follicular development with the least signals captured in primordial follicles. BMPR1B was expressed in follicular cells of all stages (Fig. 3A-D). Immunolabelling of FSHR showed no staining in follicle cells of primordial follicles but expressed in primary follicles onward (Fig. 3E-H). LHR expression was absent in both primordial (Fig. 3 I) and primary follicles, which then expressed in later stages of follicular development (Fig. 3 I-L).
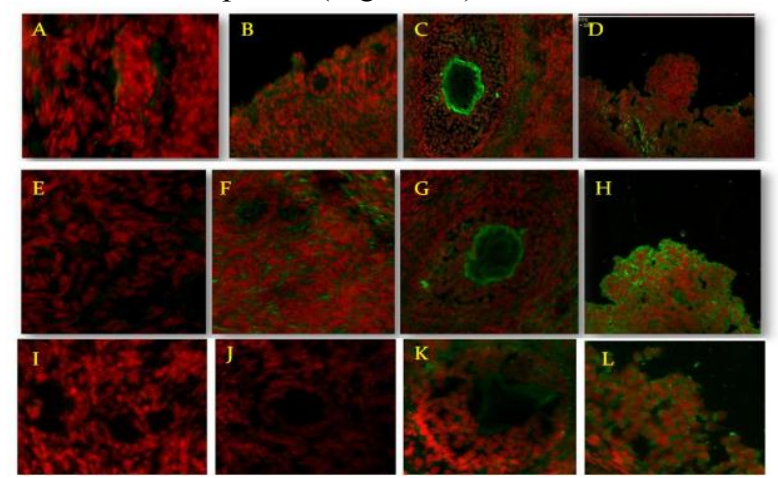

Figure 3. The expression of BMPR1b (A-D), FSHR (E-H) and LHR (I-L). A, E, I, primordial follicles; B, F, J, primary follicles; C, G, K, secondary follicles; D, H, L, Graafian follicles. Green color staining indicates a positive labeling of the receptors. E, I and J show negative staining of FSHR (E) and LHR (I, J). X40

\section{3D Image Quantification of BMP1B, FSHR, and $\angle H R$}

Expression during different follicular stages Quantitative analysis based on the intensity of fluorescent signals expressed in $3 \mathrm{D}$ images showed that the expression of BMPR1b, FSHR and LHR significantly $(\mathrm{p}<$ 0.0001) increases with follicular development (Fig. 4AC). The intensity of labeling was higher for BMPR1b and lower for LHR. BMPR-1b expression was detected in the GCs in all follicular stages of follicular development (Fig. 
4A). The FSHR expression was absent in primordial follicles but expressed in the rest stages of follicular development (Fig. 4B). LHR expression was absence in both primordial and primary follicular and expressed in the rest stages of follicular development (Fig. 4C).

A

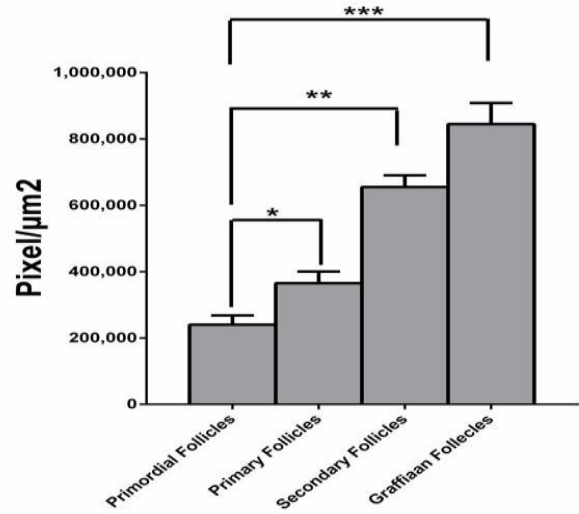

Quantification of BMPR1b in 4 follicular stages $(\mathbf{n}=\mathbf{2 0})$

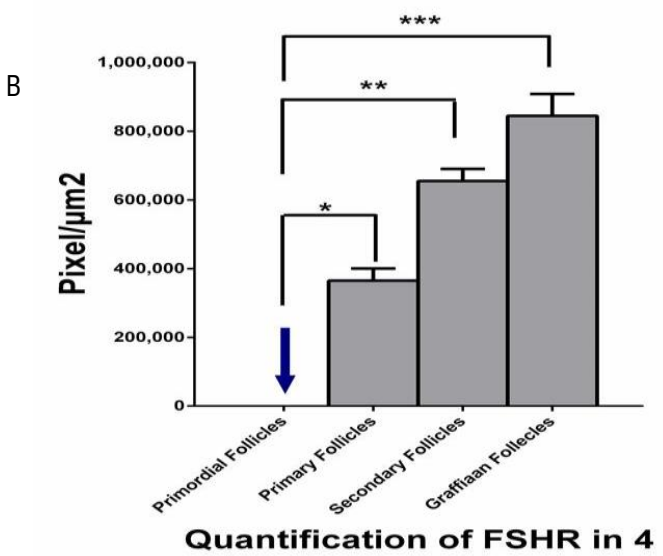
follicular stages $(\mathbf{n}=\mathbf{2 0})$

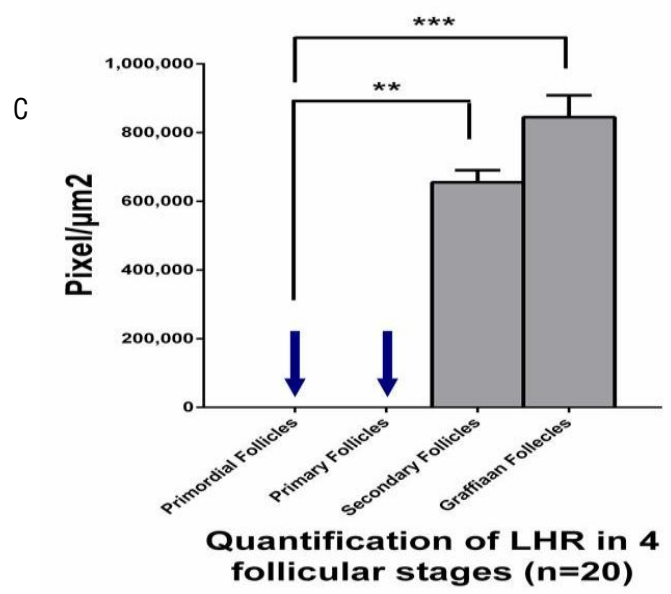

Figure 4. Showed the quantification of receptor expression $\left(\mathrm{pixel} / \mu \mathrm{m}^{2}\right)$ in 4 stages of ovarian development. A) The BMPR1b expression B)FSHR expression C) LHR expression, number of follicles $\mathrm{n}=20$.**: $\mathrm{p}<0.001, * * *: \mathrm{p}<0.0001$

\section{DISCUSSION}

This study demonstrates for the first time the temporospacial localization and quantification of BMPR1b, FSHR and LHR in sheep ovaries throughout the initial steps of folliculogenesis. The results indicate that gonadotropins are not involved in the recruitment of primordial follicles clarifying the ambiguity of the literatures [10], [11]. In addition, the in situ localization of these three receptors in the follicles across several stages of development unequivocally shows the interplay between these hormones and growth factors in the regulation of ovarian function. Traditionally, the detection and quantification of immuonoflourescent labeling is conducted by manual subjective methods presented in form of scores range from 0 (no staining) to 4 (intensive staining). This kind of studies may be subjected to bias and therefore non-reliable particularly for the purpose of statistical significance [8]. In addition, subjective approach is not efficient to detect and estimate a small amount of signals, such as within the cell membrane, within a complex structure. Furthermore, semi-quantitative method can only estimate signals emitted from the surface of a single section, which does not represent the actual amount of molecules in the $3 \mathrm{D}$ tissue structure. The present study provides alternative approach using computer-based quantitative analysis of 3D objects.

Ovarian folliculogenesis operates mainly under the control of FSH and $\mathrm{LH}$; their levels are regulated by the hypothalamic-pituitary-gonadal axis [2]. However, it has been reported that normal follicle development occurs in mice with mutation in FSH $\beta$ subunit and FSHR [12], suggesting that the regulation of initial follicle recruitment is gonadotropin-independent 13. Instead, it is now believed that the initial recruitment of primordial follicles is a gonadotropin-independent process but controlled by intraovarian factors 14 such as the BMPs 15, yet other conflicting reports indicated otherwise 10 , 16

In this study, the absence of FSHR and LHR in primordial follicles unequivocally indicates that the early stage of follicular development is gonadotropin independent. However, the precautious expression of FSHR in primary follicles suggested that FSH may be involved indirectly by up-regulating the expression of BMPR1b 6].

\section{CONUCLUSION}

The current study highlights the necessity and feasibility of using non-bias computerized method to detect and quantify the presence and distribution of a small quantity of molecules in a 3D complex structure such as the ovarian follicles. The outcome of the study is important in expanding our understanding of the role of BMPs in the regulation of ovarian functions.

\section{ACKNOWLEDGMENT}

The authors are grateful for Professor John Memo and his team and also the provision of research facilities and the scientific and technical assistance of the staff of CHIRI Biosciences Research Precinct core facility, Curtin University for their assistance to have access to and training of semi-confocal microscope and other research facilities. 


\section{REFERENCES}

[1] C. Williams and G. Erickson. (30 January, 2012). Morphology and physiology of the ovary. [Online]. Available: http://www.endotext.org/female/female1/female1.htm

[2] B. C. Fauser and A. M. Van Heusden, "Manipulation of human ovarian function: Physiological concepts and clinical consequences, "Endocr Rev, vol. 18, no. 1, pp. 71-106, 1997.

[3] S. Shimasaki, et al., "A functional bone morphogenetic protein system in the ovary," in Proc. Natl Acad Sci., U S A, vol. 96, no. 13, 1999, pp. 7282-7287.

[4] W. S. Lee, et al., "Effect of bone morphogenetic protein-7 on folliculogenesis and ovulation in the rat, "Biol. Reprod, vol. 65, no. 4, pp. 994-9, 2001.

[5] S. Shimasaki, et al., "The bone morphogenetic protein system in mammalian reproduction," Endocr Rev., vol. 25, no. 1, pp. 72-101, 2004.

[6] C. Wang and S. K. Roy, "Expression of bone morphogenetic protein receptor (BMPR) during perinatal ovary development and primordial follicle formation in the hamster: Possible regulation by FSH," Endocrinology, vol. 150, no. 4, pp. 1886-96, 2009.

[7] G. F. Erickson and S. Shimasaki, "The spatiotemporal expression pattern of the bone morphogenetic protein family in rat ovary cell types during the estrous cycle," Reprod Biol Endocrinol, vol. 1, pp. 9, 2003.

[8] K. Ilanthodi. (5th Nov, 2012). Color image analysis for staining intensity quantification - its application to medical research and $\begin{array}{lll}\text { diagnostic purposes. } & \text { Online]. Available: }\end{array}$ http://shodhganga.inflibnet.ac.in/handle/10603/5042

[9] P. F. Hall and G. Almahbobi, "Roles of microfilaments and intermediate filaments in adrenal steroidogenesis," Microsc Res Tech, vol. 36, no. 6, pp. 463-79, 1997.

[10] J. A. Flaws, et al., "Chronically elevated luteinizing hormone depletes primordial follicles in the mouse ovary," Biol Reprod, vol 57, no. 5, pp. 1233-7, 1997.

[11] S. Meredith, et al., "Unilateral ovariectomy increases loss of primordial follicles and is associated with increased metestrous concentration of follicle-stimulating hormone in old rats," BiO Reprod, vol. 47, no. 2, pp. 162-168, 1992.

[12] T. R. Kumar, M. J. Low, and M. M. Matzuk, "Genetic rescue of follicle-stimulating hormone beta-deficient mice," Endocrinology, vol. 139 , no. 7 , pp. 3289-95, 1998.
[13] R. Jaatinen, et al., "Identification of ovarian granulosa cells as a novel site of expression for bone morphogenetic protein-3 (BMP3/osteogenin) and regulation of BMP-3 messenger ribonucleic acids by chorionic gonadotropin in cultured human granulosaluteal cells," J Clin Endocrinol Metab, vol. 81, no. 11, pp. 3877-82, 1996.

[14] P. S. Tanwar, T. O. Shea, and J. R. McFarlane, "In vivo evidence of role of bone morphogenetic protein-4 in the mouse ovary," Anim Reprod Sci, vol. 106, no. 3-4, pp. 232-40, 2008.

[15] J. Massague, "TGF-beta signal transduction," Annu Rev Biochem vol. 67, pp. 753-91, 1998 .

[16] F. Otsuka, R. K. Moore, and S. Shimasaki, "Biological function and cellular mechanism of bone morphogenetic protein- 6 in the ovary," J Biol Chem, vol. 276, no. 35, pp. 32889-95, 2001.

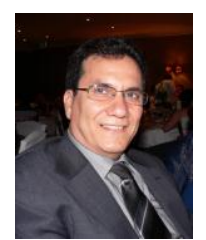

Dr. Ghanim Almhabobi has gained his $\mathrm{PhD}$ in reproductive endocrinology from University of Caen, France in 1987. His postdoctoral training was at the University of New South Wales in Sydney from 1988 to 1993 . He worked as a senior scientist at Monash University's Institute of Medical Research for 12 years before he was appointed as a Senior lecturer at Curtin University in 2005. He has obtained two NH\&MRC research grants and three other grants from Monash IVF Foundation as a chief investigator and published more than thirty papers and invited reviews in high impact factor journals within the field.

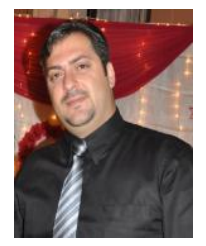

Sarmed Al-Samerria Born in Australia 1980 currently is 3rd year doctorate candidate in the school of Biomedical sciences, Curtin University, Australia. His research is under supervision of Dr. Ghanim Almahbobi. His doctorate work to investigate the role of Bone Morphogenetic protein in the regulation of ovarian function in different species using different experimental approaches. He gained a postgrad higher Diploma 2004 in medical technology and BSc (2002) in human biology and microbiology both from Baghdad University. He worked as demonstrator at institute of medical technology, Baghdad. In 2004 he served as scientist in the research centre of the radioactive and biohazard materials, ministry of technology and sciences, Iraq. Sarmed has participated in several national and international conferences and have several publications in the area of reproductive biology. 